\title{
Chemotherapy-induced Neuropathy—Where Are We Now?
}

\author{
Kathryn J Ruddy, MD, MPH, ${ }^{1}$ Neil Majithia, MD, ${ }^{2}$ Deirdre R Pachman, MD, ${ }^{3}$ Andreas S Beutler, MD ${ }^{4}$ and Charles L Loprinzi, MD \\ 1. Assistant Professor of Oncology; 2. Internal Medicine Resident; 3. Assistant Professor of Medicine and Oncology; 4. Assistant Professor of Oncology; \\ 5. Professor of Oncology, Mayo Clinic, Rochester, Minnesota, US
}

\begin{abstract}
Among the most debilitating of chemotherapy-related toxicities is neuropathy, which is associated with several commonly used oncologic agents. The specific characteristics of neurotoxicity vary between inciting drugs, with important differences in symptom characteristics and clinical course. No medications have been found to effectively prevent chemotherapy-induced neuropathy, and investigations of predictors of severe neurotoxicity to date explain only a small part of susceptibility (making it difficult to tailor chemotherapies to individual risk). Treatment has also been challenging. Although duloxetine is modestly beneficial, and Scrambler therapy shows promise in preliminary studies, no other therapies have been proved to be effective.
\end{abstract}

\section{Keywords}

Chemotherapy-related toxicities, neuropathy, chronic peripheral neuropathy, Scrambler therapy

Disclosure: Charles L Loprinzi, MD, reports that Pfizer and Competitive Technologies have provided support to Mayo for clinical trials regarding neuropathy. Kathryn J Ruddy, MD, MPH, Neil Majithia, MD, Deirdre R Pachman, MD, and Andreas S Beutler, MD, have no conflicts of interest to declare.

open Access: This article is published under the Creative Commons Attribution Noncommercial License, which permits any noncommercial use, distribution, adaptation, and reproduction provided the original author(s) and source are given appropriate credit.

Received: February 19, 2015 Accepted: February 24, 2015 Citation: Oncology \& Hematology Review, 2015;11(1):53-5 DOI: 10.17925/OHR.2015.11.01.53

Correspondence: Kathryn J Ruddy, MD, MPH, 200 First Street SW, Rochester, MN 55905, US. E: ruddy.kathryn@mayo.edu

Support: Research reported in this publication was supported by the National Institute Of Nursing Research of the National Institutes of Health (NIH) under Grant Number R01NR015259 (to ASB) and by the National Center for Advancing Translational Sciences (NCATS) of the National Institutes of Health (NIH) under Grant Numbers UL1TR000135 and KL2TR000136-09 (to KJR). The content is solely the responsibility of the authors and does not necessarily represent the official views of the NIH.

Chemotherapy-induced neuropathy (CIN), which often results from a number of commonly used oncologic drugs, including taxanes, platinum agents, bortezomib, vinca alkaloids, ixabepilone, and eribulin, is one of the most debilitating symptoms caused by chemotherapy. Most research on this toxicity has focused on paclitaxel and oxaliplatin, both of which are used frequently and can cause severe neurotoxicity.

The most widely recognized and dose-limiting aspect of CIN is a chronic peripheral neuropathy (CIPN), which commonly starts in a stocking glove distribution and is predominantly sensory in nature. These symptoms generally become progressively worse with continued treatment, and numbness and tingling are more prominent than is pain. ${ }^{1}$ When many neurotoxic chemotherapy drugs, such as taxanes, are stopped, CIPN generally begins to improve within weeks to months. ${ }^{2,3}$ However, with cisplatin and oxaliplatin, a "coasting phenomenon" may be experienced in which the neuropathy worsens during the first months off therapy. ${ }^{4}$ In the case of oxaliplatin, the slope of the worsening CIPN after treatment completion mirrors that seen during treatment, suggesting that it is not the stoppage of chemotherapy that causes the worsening symptoms, but rather the continued detrimental effect of the final dose of chemotherapy, which takes about 3 months to be fully manifested. On average, 3 months after finishing oxaliplatin, the neuropathy starts to improve. However, for some patients, CIPN can be a chronic debilitating problem for many years, regardless of the causative agent.

Less problematic is an acute, more reversible CIN that occurs with some neurotoxic agents. Oxaliplatin is the best recognized cause of acute $\mathrm{CIN}$. In contrast to the DNA damage and mitochondrial dysfunction that underlies oxaliplatin-associated chronic neuropathy, acute toxicity has been hypothesized to occur due to calcium chelation, with resultant derangements in activity of neuronal membrane ion channels. In the acute setting, neuropathy is manifested by cold sensitivity, difficulty swallowing cold liquids, throat discomfort, and muscle cramps. The severities of the first three of these symptoms are relatively well correlated in individual patients, but the degree of muscle cramps seems to be experienced as a somewhat separate entity. ${ }^{5}$ This type of neuropathy is almost universal among patients receiving oxaliplatin-based chemotherapy, with few patients completely avoiding all four types of symptoms. The severity of these symptoms after the first oxaliplatin dose is usually only half that experienced with the second dose, but symptoms tend to plateau thereafter, without any cumulative drug effect. An individual's severity of acute neuropathy in the first cycle correlates strongly with the severity of acute neuropathy 
in subsequent cycles. Although acute CIN commonly peaks at 3 days and then starts to improve, these symptoms persist to a lesser degree between 2-week oxaliplatin repeat doses. ${ }^{5}$ Substantial data illustrate that the degree of acute oxaliplatin neuropathy in individual patients predicts the degree of the more bothersome and persistent CIPN. ${ }^{5}$

Paclitaxel has long been known to cause an acute pain syndrome classically characterized as arthralgias or myalgias. However, newer data support classification of the paclitaxel-associated acute pain syndrome (P-APS) as a manifestation of acute neuropathy rather than true joint or muscle pathology. ${ }^{2,3,6}$ Although symptom severity also peaks at about 3 days after a dose of paclitaxel, the acute neuropathy symptoms of paclitaxel differ from those of oxaliplatin. The predominant complaint is pain, usually in a trunk/upper leg/hip distribution. As with oxaliplatin, the severity of acute neuropathy symptoms in the first cycle correlates with the severity of symptoms in subsequent cycles. ${ }^{2,3}$ However, as is not the case with oxaliplatin, the severity of acute neuropathy symptoms does not worsen with subsequent treatment cycles. ${ }^{2,3}$ Some data support the severity of P-APS as a predictor of severity of chronic paclitaxel neuropathy (CIPN), but not to the extent of oxaliplatin. ${ }^{5}$

CIPN susceptibility varies quite substantially from patient to patient, suggesting an underlying genetic predisposition. Patients who have Charcot-Marie-Tooth disease (CMT), a hereditary peripheral neuropathy caused by a specific mutation in one of the more than 50 different "CMT genes," are predisposed to develop severe CIPN when exposed to vincristine ${ }^{7-10}$ and occasionally have been diagnosed with CMT only after initiation of chemotherapy. ${ }^{9-15}$ CMT is thus a contraindication to neurotoxic chemotherapy. However, CMT is a rare condition, leaving the majority of CIPN cases unexplained. Moderately strong evidence exists at least partially relating these cases to other genetic variants that are not otherwise disease-causing and that were identified by genome-wide association studies ${ }^{16,17}$ or, more recently, by next-generation genome sequencing..$^{18}$ The effect size of each of these genetic variants appears comparably small, with none currently being clinically actionable.

Recognizing the magnitude of the problem posed by CIPN, extensive efforts have been directed at prevention. Recent American society of Clinical Oncology (ASCO) guidelines identified 42 randomized controlled trials that investigated agents previously hypothesized to protect against CIPN. Unfortunately, the composite data were unable to establish value for any of the agents tested in these clinical trials, making it imperative that oncology providers closely evaluate patients who receive neurotoxic chemotherapy agents. If a patient develops a moderate or worse neuropathy, it is appropriate to reassess the potential benefits of the offending chemotherapy agent in view of the ongoing toxicity.

Despite efforts to be cognizant of CIPN and to stop chemotherapy in response to worsening symptoms, many patients are left with substantial neuropathy for months to years after cessation of treatment. Recent ASCO guidelines evaluated treatment approaches for established CIPN, identifying six published randomized, controlled trials. ${ }^{19}$ The one positive recommendation from the ASCO Guideline Committee was for the use of duloxetine, a drug that has proved more effective than placebo in patients who had substantial neuropathic pain after treatment with paclitaxel or oxaliplatin..$^{20}$ However, the magnitude of benefit was quite limited. Subset analyses suggested that duloxetine worked better for neuropathy associated with oxaliplatin than with paclitaxel. An abstract of a subsequent trial, presented after the ASCO guidelines were published, provides additional confirmation that duloxetine decreases CIPN. ${ }^{21}$

In addition to duloxetine, the ASCO guidelines noted three other classes of medications reasonable to try in clinical practice despite no definitive evidence for their efficacy. One of these is the gabapentinoid class (e.g., gabapentin or pregabalin), which has been found to be beneficial for patients having other types of neuropathies. This recommendation was made even though the one placebo-controlled trial of gabapentin for CIPN was convincingly negative. ${ }^{22}$ More data are needed regarding the utility of gabapentinoids in this setting. Tricyclic antidepressants (TCAS) were also considered to be reasonable to try for CIPN based on their utility in other neuropathy states, even though only two small trials have looked at such drugs in patients with symptomatic CIPN, each failing to show any substantial improvement in symptoms. ${ }^{23,24}$ Last, a topical preparation of baclofen, amitriptyline, and ketamine was presented as a potential option based on results of one placebo-controlled trial that provided evidence suggestive of benefit. ${ }^{25}$

Efforts are ongoing to find alternative means of preventing or treating CIPN. One approach for treating chronic pain (which seems to be caused by a chronic pain signal to the brain from an area that initially was affected with acute pain) uses scrambler therapy. Scrambler therapy provides cutaneous electrostimulation to nerves that transmit pain to the brain from a particular area of the body. The electrical signals provided by Scrambler therapy are designed to create a "no pain" signal through the affected nerves, inducing the brain to change its interpretation of inputs from those nerves. It has been proposed that Scrambler therapy works similarly to spinal cord stimulation but uses cutaneous nerves to provide the requisite signal noninvasively.

In total, 12 reports of Scrambler therapy have now been published, including more than 700 patients: all 12 of these reports have been encouraging. ${ }^{26-38}$ Two relatively small randomized, controlled trials ${ }^{29,38}$ (one sham-controlled ${ }^{38}$ ) reported statistically significant reductions in chronic pain in patients receiving scrambler therapy. Three nonrandomized trials specifically supported the benefit of Scrambler therapy for treating established chronic CIPN. ${ }^{27,31,35}$ While none of the Scrambler trials were large and definitive, they, together with personal experience, suggest that a substantial proportion of patients who have chronic CIPN may benefit from this approach.

New research approaches are certainly needed to prevent or treat CIN. Improved ability to identify who will be at risk for severe neuropathy from a given chemotherapy drug would help tailor oncologic regimens and initiate early symptom management to optimize quality of life. 
1. Wolf $\mathrm{SL}$, Barton $\mathrm{DL}$, Qin R, et al., The relationship between numbness, tingling, and shooting/burning pain in patients with chemotherapy-induced peripheral neuropathy (CIPN) as measured by the EORTC QLQ-CIPN20 instrument, N06CA Support Care Cancer, 2012;20:625-32.

2. Loprinzi CL, Reeves BN, Dakhil SR, et al., Natural history of paclitaxel-associated acute pain syndrome: prospective cohort study NCCTG NO8C1, J Clin Oncol, 2011;29:1472-8.

3. Reeves BN, Dakhil SR, Sloan JA, et al., Further data supporting that paclitaxel-associated acute pain syndrome is associated with development of peripheral neuropathy: North Central Cancer Treatment Group trial N08C1, Cancer, 2012:118:5171-8.

4. Loprinzi CL, Qin R, Dakhil SR, et al., Phase III randomized, placebo-controlled, double-blind study of intravenous calcium and magnesium to prevent oxaliplatin-induced sensory neurotoxicity (NO8CB/Alliance), J Clin Oncol, 2014;32:997-1005.

5. Pachman D, Qin R, Seisler D, et al., Clinical course of patients with oxaliplatin-associated neuropathy: N08CB (Alliance), J Clin Oncol, 2014;32:3595.

6. Loprinzi CL, Maddocks-Christianson K, Wolf SL, et al., The Paclitaxel acute pain syndrome: sensitization of nociceptors as the putative mechanism, Cancer, 2007;13:399-403.

7. Dickerhoff R, Lindner W, Scheiber W, Severe vincristine neurotoxicity in a patient with Charcot-Marie-Tooth disease, Pediatr Hematol Oncol, 1988;5:61-4.

8. Graf WD, Chance PF, Lensch MW, et al., Severe vincristine neuropathy in Charcot-Marie-Tooth disease type 1A, Cancer, 1996:77:1356-62.

9. Hogan-Dann CM, Fellmeth WG, MCGuire SA, et al. Polyneuropathy following vincristine therapy in two patients with Charcot-Marie-Tooth syndrome, JAMA, 1984;252:2862-3.

10. Naumann R, Mohm J, Reuner U, et al., Early recognition of hereditary motor and sensory neuropathy type 1 can avoid life-threatening vincristine neurotoxicity, $\mathrm{Br} J \mathrm{Haematol}$, 2001;115:323-5.

11. Mercuri E, Poulton 」, Buck 」, et al , Vincristine treatment revealing asymptomatic hereditary motor sensory neuropathy type 1A. Arch Dis Child, 1999:81:442-3.

12. Nakamura T, Hashiguchi A, Suzuki $S$, et al., Vincristine exacerbates asymptomatic Charcot-Marie-Tooth disease with novel EGR2 mutation, Neurogenetics, 2012;13:77-82.

13. Neumann $Y$, Toren $A$, Rechavi $G$, et al., Vincristine treatment triggering the expression of asymptomatic Charcot-Marie-Tooth disease, Med Pediatr Oncol, 1996;26:280-3.
14. Olek MJ, Bordeaux B, Leshner RT, Charcot-Marie-Tooth disease type I diagnosed in a 5-year-old boy after vincristine neurotoxicity, resulting in maternal diagnosis, J Am Osteopath AsSOC, 1999:99:165-7.

15. Chauvenet AR, Shashi V, Selsky C, et al., Vincristine-induced neuropathy as the initial presentation of Charcot-Marie-Tooth disease in acute lymphoblastic leukemia: a Pediatric Oncology Group study, J Pediatr Hematol Oncol, 2003;25:316-20.

16. Saif MW, Lee AM, Offer SM, et al., A DPYD variant (Y186C) specific to individuals of African descent in a patient with lifethreatening 5-FU toxic effects: potential for an individualized medicine approach, Mayo Clin Proc, 2014:89:131-6.

17. Baldwin RM, Owzar K, Zembutsu H, et al., A genome-wide association study identifies novel loci for paclitaxel-induced sensory peripheral neuropathy in CALGB 40101, Clin Can Res, 2012;18:5099-109.

18. Beutler AS, Kulkarni AA, Kanwar R, et al., Sequencing of Charcot-Marie-Tooth disease genes in a toxic polyneuropathy, Ann Neurol, 2014:76:727-37.

19. Hershman DL, Lacchetti C, Dworkin RH, et al Prevention and management of chemotherapy-induced peripheral neuropathy in survivors of adult cancers: American Society of Clinical Oncology clinical practice guideline, J Clin Oncol, 2014;32:1941-67.

20. Smith EM, Pang H, Cirrincione $\mathrm{C}$, et al., Effect of duloxetine on pain, function, and quality of life among patients with chemotherapy-induced painful peripheral neuropathy: a randomized clinical trial, JAMA, 2013:309:1359-67.

21. Hirayama $Y$, Terui T, Koike $K$, et al. Effect of duloxetine on chemotherapy-induced painful peripheral neuropathy, I Clin Oncol, 2014;32(Suppl. 31):abstr. 181.

22. Rao RD, Michalak JC, Sloan JA, et al., Efficacy of gabapentin in the management of chemotherapy-induced peripheral neuropathy: a phase 3 randomized, double-blind, placebocontrolled, crossover trial (NOOC3), Cancer, 2007;110:2110-8.

23. Hammack JE, Michalak JC, Loprinzi CL, et al., Phase III evaluation of nortriptyline for alleviation of symptoms of cis-platinuminduced peripheral neuropathy Pain 2002:98:195-203.

24. Kautio AL, Haanpaa M, Saarto T, et al., Amitriptyline in the treatment of chemotherapy-induced neuropathic symptoms, J Pain Symptom Manage, 2008;35:31-9.

25. Barton DL, Wos EJ, Qin R, et al., A double-blind, placebocontrolled trial of a topical treatment for chemotherapy-induced peripheral neuropathy: NCCTG trial N06CA, Support Care Cancer, 2011:19:833-41.
26. Abdi S, Lakkimsetty VM, Barrera J, et al., The use of "Scrambler therapy" for failed back surgery syndrome, Pain Physician, 2011;14:E465-91.

27. Coyne PJ, Wan W, Dodson P, et al., A trial of Scrambler therapy in the treatment of cancer pain syndromes and chronic chemotherapy-induced peripheral neuropathy, J Pain Pall Care Pharmacother, 2013;359-64.

28. Ko YK, Lee HY, Lee WY, Clinical experiences on the effect of Scrambler therapy for patients with postherpetic neuralgia, Korean J Pain, 2013;26:98-101.

29. Marineo G, Iorno V, Gandini C, et al., Scrambler therapy may relieve chronic neuropathic pain more effectively than guideline-based drug management: results of a pilot, randomized, controlled trial, J Pain Symptom Manage, 2012;43:87-95

30. Moon JY, Kurihara C, Beckles JP, et al., Predictive factors associated with success and failure for Calmare (Scrambler) therapy: a multi-center analysis, Clin J Pain, 2014 [Epub ahead of print].

31. Pachman DR, Weisbrod BL, Seisler DK, et al., Pilot evaluation of Scrambler therapy for the treatment of chemotherapy-induced peripheral neuropathy, Suppor Care Cancer, 2015;23:943-51.

32. Park HS, Sin WK, Kim HY, et al., Scrambler therapy for patients with cancer pain—case series, Korean J Pain, 2013;26:65-71.

33. Ricci M, Pirotti S, Scarpi E, et al., Managing chronic pain: results from an open-label study using MC5-A Calmare ${ }^{\circledR}$ device, Support Care Cancer, 2011:20:405-12.

34. Sabato AF, Marineo G, Gatti A, Scrambler therapy, Minerva Anestesiol, 2005:71:479-82.

35. Smith TJ, Coyne PJ, Parker GL, et al., Pilot trial of a patientspecific cutaneous electrostimulation device (MC5-A Calmare ${ }^{\oplus}$ ) for chemotherapy-induced peripheral neuropathy, J Pain Symptom Manage, 2010;40:883-91.

36. Smith TJ, Marineo G, Treatment of postherpetic pain with Scrambler therapy, a patient-specific neurocutaneous electrical stimulation device, Am J Hosp Pall Care, 2013 [Epub ahead of printl

37. Sparadeo F, Kaufman C, D'Amato S, Scrambler therapy: an innovative and effective treatment for chronic neuropathic pain, J Life Care Plan, 2012;11:3-15.

38. Starkweather AR, Coyne P, Lyon DE, et al., Decreased low back pain intensity and differential gene expression following Calmare ${ }^{\oplus}$ results from a double-blinded randomized shamcontrolled study, Res Nurs Health, 2015;38:29-38. 